Technical Note

\title{
Improved sampling techniques for the direct simulation Monte Carlo method
}

\author{
Quanhua Sun ${ }^{\mathrm{a}, *}$, Jing Fan ${ }^{\mathrm{a}}$, Iain D. Boyd ${ }^{\mathrm{b}}$ \\ ${ }^{a}$ Key Laboratory of High Temperature Gas Dynamics, Institute of Mechanics, Chinese Academy of Sciences, Beijing 100190, China \\ ${ }^{\mathrm{b}}$ Department of Aerospace Engineering, University of Michigan, Ann Arbor, MI 48109, USA
}

\section{A R T I C L E I N F O}

\section{Article history:}

Received 20 September 2007

Received in revised form 18 April 2008

Accepted 22 April 2008

Available online 1 May 2008

\begin{abstract}
A B S T R A C T
The direct simulation Monte Carlo (DSMC) method is a widely used approach for flow simulations having rarefied or nonequilibrium effects. It involves heavily to sample instantaneous values from prescribed distributions using random numbers. In this note, we briefly review the sampling techniques typically employed in the DSMC method and present two techniques to speedup related sampling processes. One technique is very efficient for sampling geometric locations of new particles and the other is useful for the Larsen-Borgnakke energy distribution.
\end{abstract}

(c) 2008 Elsevier Ltd. All rights reserved.

\section{Introduction}

The direct simulation Monte Carlo (DSMC) method is a particle simulation method that aims to calculate practical gas flows through computations of motions and collisions of modeled molecules [1]. It is the most successful method for rarefied gas flow simulations, and has been applied to many fields [2,3]. These include the traditional field of rarefied atmospheric gas dynamics, the emerging field of micro-fluidics, and very-low pressure fields such as space propulsion, material processing, and vacuum systems. It is expected that applications of the DSMC method will continue to expand as the computational resources become more and more affordable and efficient.

As a Monte Carlo method, the DSMC method employs distribution functions to generate representative particles and perform particle collisions. Sampling from a prescribed distribution is one of the major tasks for the DSMC method. The commonly used sampling techniques are the inverse-cumulative method and the acceptance-rejection method. Both methods employ random numbers between zero and one to realize the distribution. However, the inverse-cumulative method is not general and the acceptancerejection method is usually expensive. As Bird [1] pointed out: special methods are more efficient than commonly used sampling techniques. Nevertheless, sampling techniques have been seldom discussed. Only recently, several efficient acceptance-rejection methods have been proposed for the Maxwellian inflow distribution [4,5]. As the DSMC method becomes more and more popular, it is necessary to develop techniques to improve the efficiency of sampling processes involved in DSMC simulations.

In this note, we briefly review the general sampling techniques employed in typical DSMC simulations and propose two techniques to improve the sampling efficiency for some distributions.

\footnotetext{
* Corresponding author. Tel.: +8610 82544023; fax: +8610 62561284

E-mail address: qsun@imech.ac.cn (Q. Sun).
}

\section{Sampling techniques employed in DSMC}

The direct simulation Monte Carlo method employs individual particles to mimic flow details. Every simulated particle usually represents a large number of real molecules. The particles own several properties that change during the simulation following physical distributions. These properties include the position, velocity, and internal energy. In general, particles are introduced to the simulation domain during initialization process and through computational boundaries. Then the number of simulated particles and the properties of each particle are to be determined statistically. Later particles adjust their positions in a deterministic way during the convection step whereas collisions update the velocities and internal energies of particles statistically. When the statistics is involved, physical distributions are sampled to set or adjust the properties of the particles. The current commonly employed sampling techniques are the inverse-cumulative method and the acceptance-rejection method.

The inverse-cumulative method samples an instantaneous value $x$ from the distribution $f(x)$ using the cumulative distribution function $F(x)$, which is defined as

$F(x)=\int_{a}^{x} f(x) \mathrm{d} x$

where $x \in[a, b)$. This approach solves Eq. (2) using a random number $R_{f}$ whose value is between 0 and 1 , which is illustrated in Fig. 1 [1]. It is a very fast method

$F(x)=R_{f}$

if an explicit expression for $x$ could be derived. Otherwise, the Newton iteration method may be used since $F(x)$ is a monotonic function:

$x_{n+1}=x_{n}-\frac{F\left(x_{n}\right)-R_{f}}{f\left(x_{n}\right)}$ 


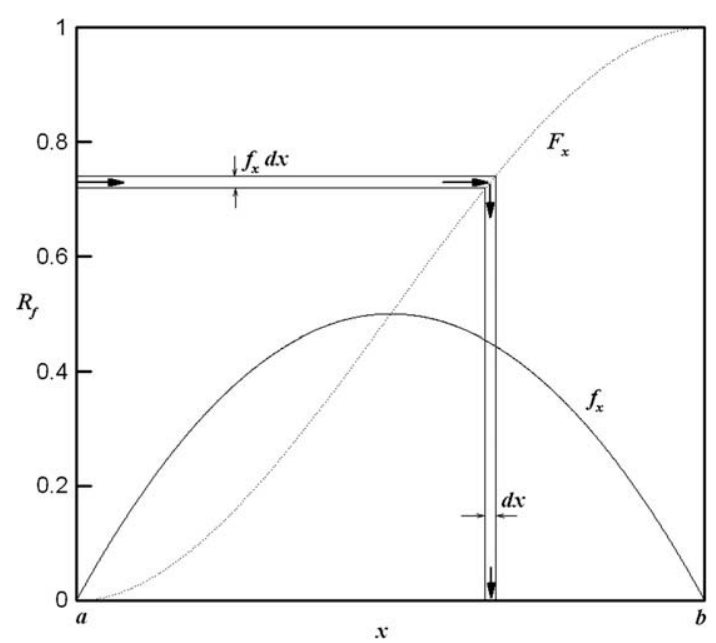

Fig. 1. Illustration of the inverse-cumulative method.

However, it may take many iterations to obtain the solution with a good accuracy, which can be expensive when a lot of samples have to be made.

The acceptance-rejection method is a general method for sampling a function. It requires to find an envelope function, $e(x)$, whose value is larger than the prescribed function $f(x)$ :

$e(x) \geqslant f(x), \quad x \in[a, b)$

There are two steps employed in this approach: (1) sample a value of $x$ from function $e(x)$; (2) if the value of $f(x) / e(x)$ is larger than a random number $R_{f}$, accept $x$; otherwise repeat step (1). In general, a value of $x$ should be easily sampled from function $e(x)$. In addition, a high acceptance ratio for step (2) is desired, which means that the envelope function should be close to the original distribution function $f(x)$. For many situations, a good envelope function is not easy to find. A simple choice is to choose the maximum value of $f(x)$ as a constant function (see Fig. 2). However, the value of $f(x) / e(x)$ especially $f(x) / f_{\max }$ can be very small for $x$ in a certain range, which makes the acceptance ratio very small. For the efficiency purpose, it is acceptable to limit the range of $x$ during step (1) as long as the cutoff error is negligible.

In DSMC simulations, the inverse-cumulative method is generally employed when an explicit expression for a distribution is

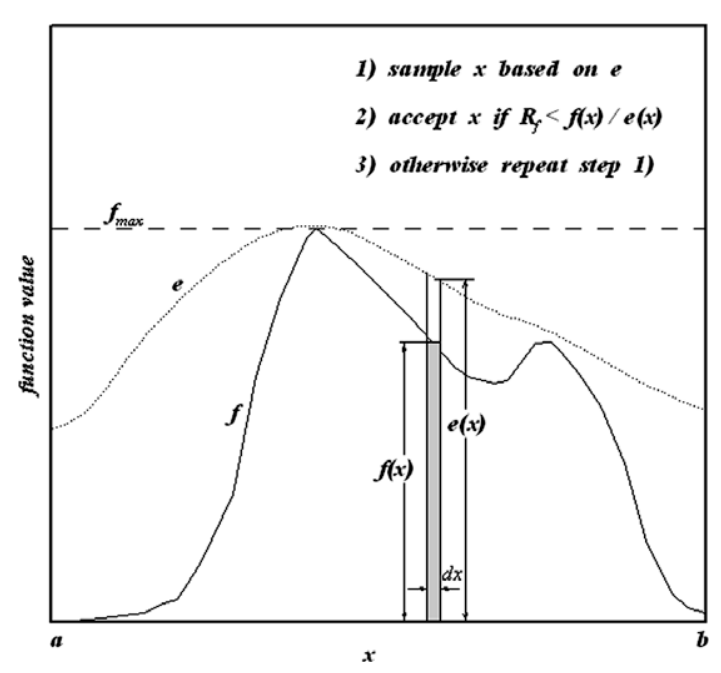

Fig. 2. Illustration of the acceptance-rejection method. available. These scenarios include sampling velocities from Maxwellian distributions and setting the accommodated reflected velocities for particle-surface interactions, particle positions from Cartesian meshes, and the internal energy for initial particles having two degrees of freedom for the internal energy. The acceptance-rejection method is employed for sampling from other distributions, such as Larsen-Borgnakke distributions for energy re-distributing and uniform distribution for geometric locations. These two methods are typically used in a straightforward way. However, modifications can be made to improve the sampling efficiency for some distributions.

\section{Improved geometric sampling technique}

Each newly generated particle is assigned a geometric location within the computational domain. The inverse-cumulative method can be employed when the location is sampled from a line or when the mesh is strictly rectangular. For general meshes, the acceptance-rejection method is commonly used. The procedure for sampling a multi-dimensional location consists of two steps as illustrated in Fig. 3: (1) find uniformly a location in an enclosed geometry box such as abcda; (2) accept this location if it is located within the given geometry such as 12345 ; otherwise, repeat step (1). The time cost for this approach depends strongly on the shape of the geometry. One is due to the acceptance ratio, the area or volume ratio of the geometry to the enclosed box; and the other is the cost to determine whether the location is within the geometry or not. Our experience shows that over 100 attempts can be encountered in simulations when the acceptance ratio is small for cases such as one in Fig. 3b. To improve the sampling efficiency, we propose a direct sampling approach for 2D and 3D simulations.

The direct sampling approach takes advantage of the geometry when selecting a location. The location is selected by sampling an element from the geometry-enclosed area. To illustrate this approach, we sample a location within the triangle $a b c$ as shown in Fig. 4:

(1) Select randomly a location $d$ on line $a b$ :

$$
\vec{r}_{d}=\vec{r}_{a}+R_{f 1}\left(\vec{r}_{b}-\vec{r}_{a}\right)
$$

Clearly, the area of the shadowed triangle $c d$ remains constant when location $d$ varies along the line.

(2) Select randomly a location $e$ on line $c d$ based on the cutting area as shown in Fig. 4a:

$$
\vec{r}_{e}=\vec{r}_{c}+\sqrt{R_{f 2}}\left(\vec{r}_{d}-\vec{r}_{c}\right)
$$

Here the location $e$ is the sampled location. This approach does not involve any attempt and there is no need to verify particle's location. Hence, this approach is very efficient and is strongly recommended. An example of the distribution of 10,000 calls using this approach is shown in Fig. 4b, which clearly shows the uniformity of the distribution. For 3D tetrahedral cells, a third step is added:

$\vec{r}_{f}=\vec{r}_{t}+\sqrt[3]{R_{f 3}}\left(\vec{r}_{e}-\vec{r}_{t}\right)$

where $t$ is the fourth node forming the cell, and $\vec{r}_{f}$ is the sampled location. It seems that this approach cannot be applied to axi-symmetric cases though.

For general 2D or 3D cell shapes, they can be divided into triangles or tetrahedrons. An example is shown in Fig. 5 for 2D cases. The areas of every triangle can be pre-calculated, and their cumulative percentage of area in the entire cell is also calculated. Then a triangle is selected using a random number as shown in Fig. 5. 
a

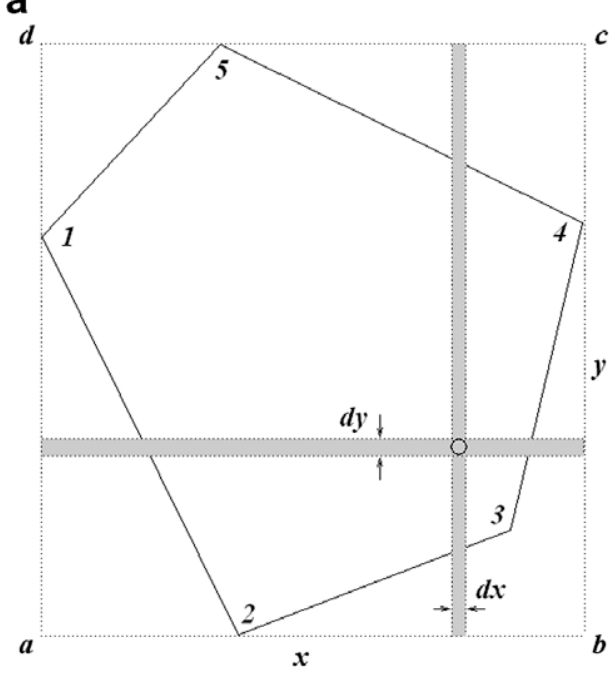

b

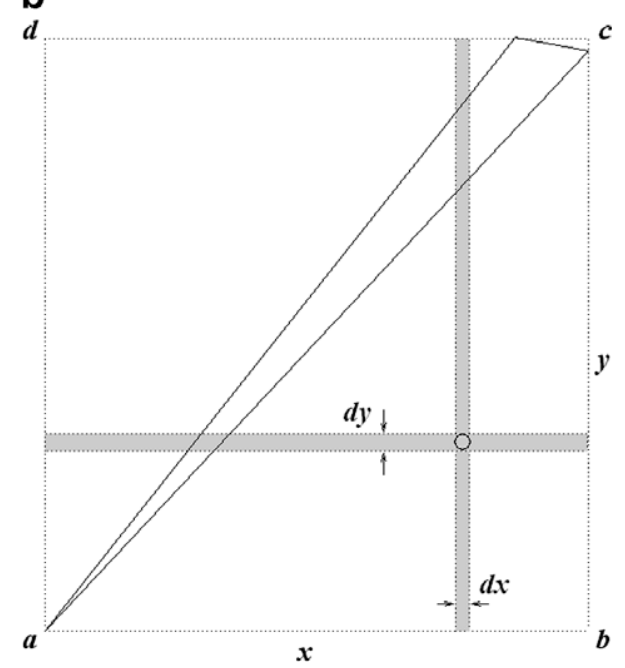

Fig. 3. Geometric location sampling using the acceptance-rejection method: (a) typical case and (b) inefficient case.

a

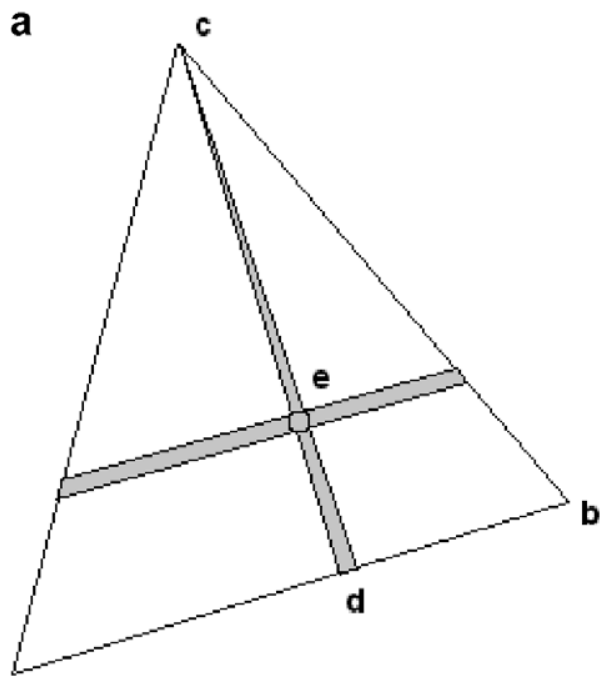

b

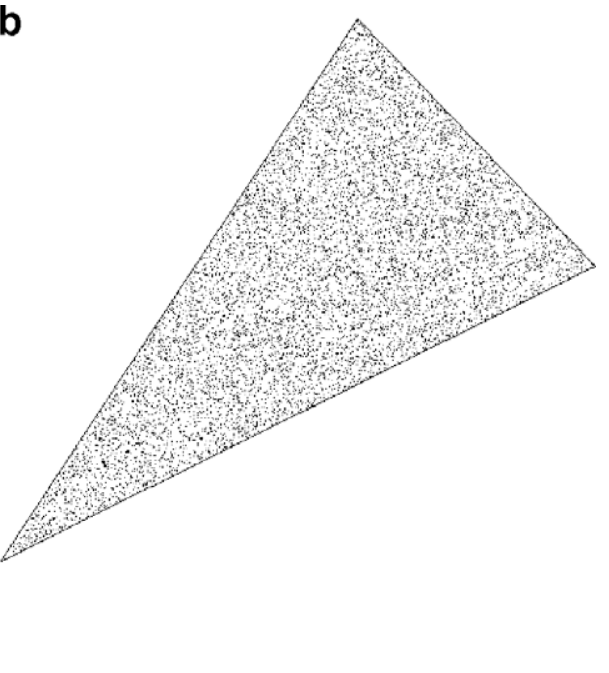

a

Fig. 4. Geometric location sampling using the direct sampling approach: (a) illustration and (b) numerical example.

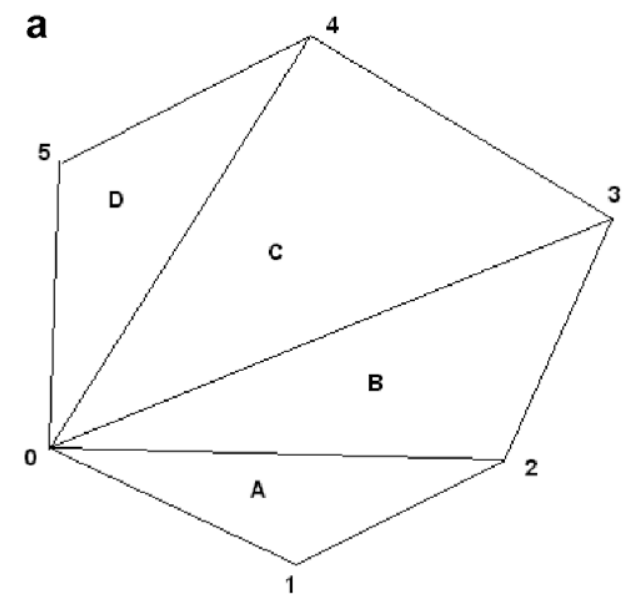

b

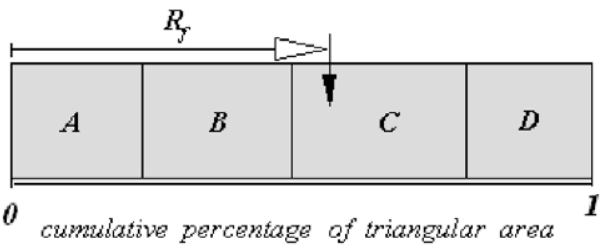

Fig. 5. Illustration of selecting a triangle from a polygon: (a) division and (b) selection. 
After a triangle is selected, the location is sampled using the proposed direct sampling approach. Note that for rectangular cells, a particle's location can be easily sampled using the inverse-cumulative method and there is no need to break them into triangular cells.

\section{Improved sampling technique for the Larsen-Borgnakke distribution}

Particle collisions are a key part of the DSMC method. Collisions usually result in energy re-distribution between colliding particles. The energy exchange is usually modeled using the Larsen-Borgnakke phenomenological model [1]. This model employs the equal-partition principle. The distribution of energy $E_{a}$ from a total of $E_{a}+E_{b}$ is expressed as Eq. (8):

$f(x)=f\left(\frac{E_{a}}{E_{a}+E_{b}}\right)=\frac{\Gamma\left(\xi_{a}+\xi_{b}\right)}{\Gamma\left(\xi_{a}\right) \Gamma\left(\xi_{b}\right)} x^{\xi_{a}-1}(1-x)^{\xi_{b}-1}$

where $\xi_{a}$ and $\xi_{b}$ are the numbers of degrees of freedom for $E_{a}$ and $E_{b}$, respectively. The instantaneous value of energy $E_{a}$ can be obtained using the acceptance-rejection method. Usually, the envelope function is fixed at the maximum value of $f(x)$. Then the acceptance rate is derived as

$\frac{f(x)}{f_{\max }}=\left(\frac{\xi_{a}+\xi_{b}-2}{\xi_{a}-1} x\right)^{\xi_{a}-1}\left(\frac{\xi_{a}+\xi_{b}-2}{\xi_{b}-1}(1-x)\right)^{\xi_{b}-1}$

However, many attempts have to be made to sample a value when $\xi_{a}$ and $\xi_{b}$ are not of the same order of magnitude [6]. Here we propose a remedy to avoid finding $x$ itself by converting $x$ to a new variable $y$ whose statistical value is close to 0.5 . The transformation is set as $y=x^{1 / a}$, where $a=\log _{2}^{\left(\breve{\xi}_{a}+\breve{\zeta}_{b}\right) / \check{\xi}_{a}}$. Then the distribution for $y$ is

$f(y)=\frac{a \Gamma\left(\xi_{a}+\xi_{b}\right)}{\Gamma\left(\xi_{a}\right) \Gamma\left(\xi_{b}\right)}\left(y^{a}\right)^{\xi_{a}-1 / a}\left(1-y^{a}\right)^{\xi_{b}-1}$

and the acceptance ratio is

$\begin{aligned} \frac{P}{P_{\max }}(y) & =\left(\frac{\xi_{a}+\xi_{b}-1-1 / a}{\xi_{a}-1 / a} y^{a}\right)^{\xi_{a}-1 / a} \\ & \times\left(\frac{\xi_{a}+\xi_{b}-1-1 / a}{\xi_{b}-1}\left(1-y^{a}\right)\right)^{\xi_{b}-1}\end{aligned}$
To compare the numerical performance of the standard and modified acceptance-rejection procedure for the Larsen-Borgnakke model, the average number of attempts required during the acceptance-rejection procedure is plotted in Fig. 6a where the percentage of one energy in the total is $E_{a} /\left(E_{a}+E_{b}\right)$. Clearly, the modified procedure improves greatly the numerical efficiency when $\xi_{a}$ is much smaller than $\xi_{b}$, which is usually the case for complicated molecules such as clusters having many atoms [6]. Numerical tests also show that the commonly used procedure can accept unusual values with a relatively large probability. For example, Fig. 6b shows the sample history of the energy percentage $\left(E_{a} /\left(E_{a}+E_{b}\right)\right)$ whose theoretical mean value is $2 \mathrm{e}^{-4}$. We find that there are four values larger than 0.1 among 10,000 values, which is statistically too much. These undesired values, however, are avoided when the modified procedure is employed (the plot is otherwise similar to Fig. 6b, and is therefore not shown).

\section{Concluding remarks}

The direct simulation Monte Carlo method heavily involves sampling a value from various distributions. Most of the distributions followed by the DSMC method can be realized using explicit expressions with the help of random numbers whose values are within 0 and 1 . Particularly we proposed an efficient direct sampling approach to sample geometric locations for particles. The acceptance-rejection method is also required for some distributions. One is the Maxwellian inflow distribution for which Garcia and Wagner has proposed several efficient envelope functions. Another is the energy redistribution function of the Larsen-Borgnakke model. We proposed a transformation that could improve the acceptance rate when using the acceptance-rejection method. As the DSMC method undergoes various developments and detailed implementation may vary from package to package, development of sampling techniques for specific distributions may improve the performance of DSMC simulations.

\section{Acknowledgement}

This work was partially supported by the National Natural Science Foundation of China through Grant No. 10742001.
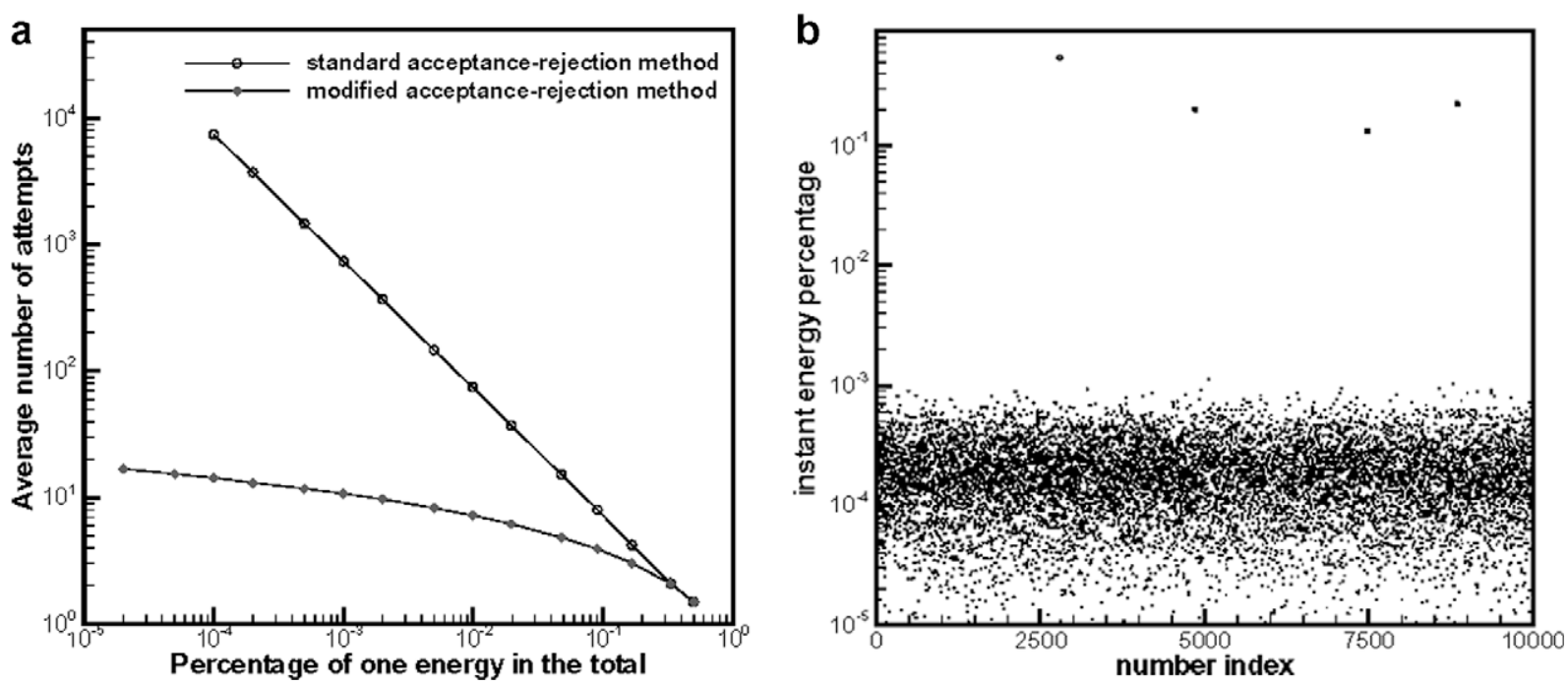

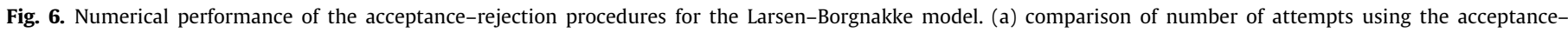
rejection procedure and (b) instantaneous distribution of the energy percentage using the standard acceptance-rejection method. 


\section{References}

[1] Bird GA. Molecular gas dynamics and the direct simulation of gas flows. Oxford University Press; 1994.

[2] Oran ES, Oh CK, Cybyk BZ. Direct simulation Monte Carlo: recent advances and applications. Ann Rev Fluid Mech 1998;30:403-41.

[3] Liou W, Fang Y. Microfluid mechanics: principles and modeling. McGraw Hill; 2005.
[4] Lilley CR, Macrossan MN. Methods for implementing the stream boundary condition in DSMC computations. Int J Num Meth Fluids 2003;42: 1363-71.

[5] Garcia AL, Wagner W. Generation of the Maxwellian inflow distribution. J Comput Phys 2006;217:693-708.

[6] Sun Q Boyd ID, Tatum KE. Particle simulation of gas expansion and condensation in supersonic jets. AIAA 2004:2004-587. 